\title{
Expression of Leptin and Leptin Receptor Messenger Ribonucleic Acids in Thyroid Carcinoma
}

\author{
Katsuhiro T*, Mai S, Shiori S, Ryohei O, Wataru S \\ and Yusuke $O$ \\ Department of Breast and Thyroid Surgery, Kawasaki \\ Medical School, Japan \\ *Corresponding author: Katsuhiro Tanaka, \\ Department of Breast and Thyroid Surgery, Kawasaki \\ Medical School, 701-0192, Japan
}

Received: J anuary 25, 2018; Accepted: February 19, 2018; Published: February 28, 2018

\begin{abstract}
A high Body Mass Index (BMI) is a risk factor for thyroid cancer. We evaluated expression levels of Leptin and Leptin-R in thyroid cancer using real-time PCR, and analyzed the correlations between clinical features and each expression level. The subjects of this study were 42 thyroid carcinoma patients (38 papillary carcinoma and 4 poorly differentiated) who underwent surgery in our hospital. Leptin and Leptin-R were detected in all patients. There was a significant relationship between the ratio of Leptin Ct value and reference ( $\beta$-actin) Ct value and $B M I(R=0.39, p<0.01)$. Furthermore, there was a significant relationship between the ratio of Leptin-R Ct value and reference $C t$ value and $B M I(R=0.42$, $p<0.005)$. There were no significant relationships among the ratios of Leptin and Leptin-R and age, gender, tumor size, lymph node involvement, extra thyroidal infiltration, recurrence and survival. In thyroid cancer, higher Leptin and Leptin-R expression correlate significantly with high BMI.
\end{abstract}

Keywords: Thyroid carcinoma; Leptin; Body mass index

\section{Introduction}

Thyroid carcinoma is the most common malignancy of the endocrine organs. Several factors are reported to initiate thyroid carcinoma. A higher Body Mass Index (BMI) is as one such factor [1]. Thyroid hormone generally never acts on follicular cells autocrine receptors, but reduces the body weight by controlling the metabolism of each organ. Concerning BMI, Leptin is a well-known factor involved in the regulation of body weight and is an important mediator of obesity. Circulating Leptin acts as a regulator of food intake from hypothalamic-mediated effects via Leptin Receptor (Leptin-R) [2]. Six splice variants of Leptin-R have been identified to date; a long isoform (Leptin- $\mathrm{Rb}$ ), four short isoforms discriminated by the different intracellular domain lengths (Leptin-Ra, c, d, f), and the secreted isoform (Leptin-Re), which modulates the action of Leptin [3].

The concentration of serum Leptin in patients with hypothyroidism is higher than that in normal subjects, while that in hyperthyroidism is lower [4]. The serum Leptin is secreted mainly from adipose tissue and affects malignant tumors such as breast, colorectal, prostate, pancreatic, ovarian and lung cancers [5]. Leptin and Leptin-R expression have also been studied in the context of thyroid cancers. In a study of papillary thyroid carcinoma patients, Leptin-R and Leptin were found to be expressed in $80.1 \%$ (410/512) and $49.1 \%$ (252/513), respectively, and Leptin-R expression was strongly associated with aggressive clinical and pathological features [6]. However, in another study, Leptin-R and Leptin were detected in $51.0 \%$ ( 25 of 49 ) and $36.7 \%$ (18 of 49 ) of cases, respectively, and showed no significant association with clinical features except for tumor size and lymph node involvement [7]. The significance of the expression of Leptin-R and Leptin in thyroid carcinomas is thus still controversial. Therefore, we elucidated the expression level of Leptin-R and Leptin in thyroid carcinomas using quantitative Polymerase Chain Reaction (PCR) and demonstrated the relationship between clinical features and both expression levels.

\section{Materials and Methods}

\section{Materials}

The subjects of this study were 42 thyroid carcinoma patients ( 38 papillary carcinoma and 4 poorly differentiated type) who underwent surgery in our hospital. All patients underwent dissection of cervical lymph nodes in addition to thyroidectomy. For this study, we used fresh frozen sections of the primary tumor from each patient that were resected at each initial surgical procedure. The thyroid function of all the patients was euthyroid before each primary operation. The BMI of each patient was obtained a few days before operation.

Informed consent was obtained from all enrolled patients. This study protocol was reviewed and approved by Kawasaki Medical School ethics committee (No. 2353).

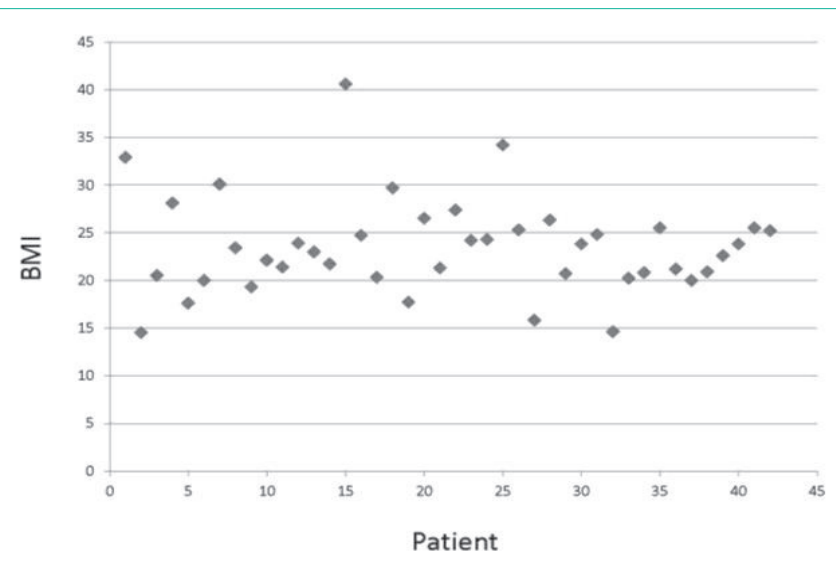

Figure 1: Distribution of BMI.

The distribution of BMI of each patient is shown. The average BMI was $23 \pm 5.4$.
Annals Thyroid Res - Volume 4 Issue 1 - 2018

Submit your Manuscript | www.austinpublishinggroup.com

Katsuhiro et al. () All rights are reserved
Citation: Katsuhiro T, Mai S, Shiori S, Ryohei O, Wataru S and Yusuke O. Expression of Leptin and Leptin Receptor Messenger Ribonucleic Acids in Thyroid Carcinoma. Annals Thyroid Res. 2018; 4(1): 126-129. 


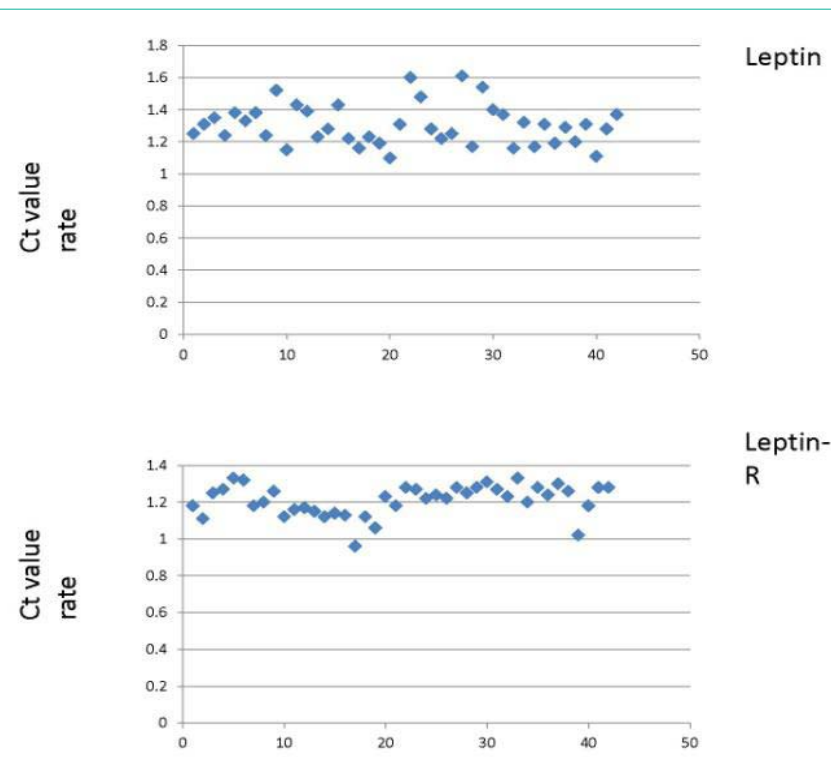

Figure 2: Average ratio of $\mathrm{Ct}$ value

The average ratios of $\mathrm{Ct}$ values of leptin versus reference (Figure $2 \mathrm{a}$ ) and leptin- $R$ versus reference (Figure $2 b$ ) were $1 \pm 0.08$ and $1 \pm 0.07$, respectively.

\section{Real-time PCR}

Total cellular RNA was extracted from microdissected snapfrozen tissues using the phenol-guanidine-isothiocyanate method with Trizol ${ }^{\circledR}$ Plus RNA Purification Kit (Life Technologies Tokyo, Japan) according to the manufacturer's protocol. The purity of the total RNA was assessed by the ratio of optical density at 260 $\mathrm{nm}$ to $280 \mathrm{~nm}$ (acceptable values being between 1.6 and 1.9). One microgram of total RNA and 20 pmol oligo dT primer in $12.5 \mathrm{ml}$ Diethyl Pirocarbonate (DEPC)- treated water were heated to $70{ }^{\circ} \mathrm{C}$ for $2 \mathrm{~min}$, followed by cooling on ice for $1 \mathrm{~min}$. cDNA synthesis was initiated using $200 \mathrm{U}$ recombinant Moloney-murine leukaemia virus reverse transcriptase (Clontech Lab. Inc., Palo Alto, CA, USA) under conditions recommended by the manufacturer.

Detection of Leptin and Leptin-R by real-time PCR was performed on a 7500 Real Time PCR System (Applied Biosystems, Tokyo, Japan). Amplification was achieved in a $20 \mu$ l volume containing $2 \mu \mathrm{l}$ cDNA, $5 \mathrm{nM}$ of each primer, $2.5 \mathrm{nM}$ of each Taqman ${ }^{\circledR}$ probe (Toyobo Lifescience, Osaka, Japan), and THUNDERBIRD probe qPCR Mix ${ }^{\oplus}$ (Toyobo Lifescience, Osaka, Japan) according to the manufacturer's protocol. The reaction mixture was subjected to 40 cycles of amplification followed by post-PCR Fluorescence Melting Curve Analysis (FMCA). The forward primer for Leptin was 5'-AGA GTG GCT TAG AGG AGT CAG-3' and that for Leptin-R was 5'TCC AGT CAC TCC AGA TTT CCT-3', and the reverse primer for Leptin was 5'-TGGCTTCCAGGTATCTCCA-3' and for Leptin-R was 5'-CAGAGAATTCTACAACAGTTATCAGAG-3'. The Taqman $^{\circ}$ probe for Leptin was 5'-/56-FAM/TGAAGAGCA/ZEN/ TTGCATGGACACCCC/31ABkFQ/-3' and for Leptin-R was 5'-/56FAM/CAGTCTCTT/ZEN/GCCCCTCACCTGAAC/31ABkFQ/-3'.

We calculated the ratio of threshold cycle $(\mathrm{Ct})$ values of Leptin and Leptin-R and the $\mathrm{Ct}$ reference value ( $\beta$-actin) for each specimen. In addition, we evaluated the relationships between the ratios in each patient and their clinical characteristics.
Table 1: Characteristics of enrolled patients.

\begin{tabular}{|c|c|c|}
\hline Age $(\mathrm{y})^{\star}$ & & 53 \\
\hline \multirow{2}{*}{ Gender } & $\mathrm{M}$ & $10(23.8 \%)$ \\
\cline { 2 - 3 } & $\mathrm{F}$ & $32(76.2 \%)$ \\
\hline Tumor size (cm)• & & 3.0 \\
\hline \multirow{2}{*}{$\mathrm{pEX}$} & & $30(71.4 \%)$ \\
\hline \multirow{2}{*}{$\mathrm{pN}$} & + & $12(28.6 \%)$ \\
\cline { 2 - 3 } & - & $6(14.3 \%)$ \\
\cline { 2 - 3 } & + & $36(85.7 \%)$ \\
\hline
\end{tabular}

-median, Parenthesis means percentage.

\section{Statistical analysis}

As the post-hoc test, Spearman's rank correlation test was used, and $\mathrm{p}<0.05$ was considered significant. All statistical analyses were performed with EZR (Saitama Medical Center, Jichi Medical University), which is a graphical user interface for $\mathrm{R}$ (The $\mathrm{R}$ Foundation for Statistical Computing, version 2.13.0).

\section{Results and Discussion}

\section{Patient Characteristics (Table 1)}

The median age and primary tumor size were 53 years and 3.0 $\mathrm{cm}$, respectively. Thirty-two of the patients were women (76.2\%), and 10 were men $(23.8 \%)$. There were 12 patients $(28.6 \%)$ with positive pEX (infiltrating to adjacent organs other than muscles). Pathological lymph node involvement (central and/or lateral cervix) was detected in 36 patients $(85.7 \%)$. The average BMI was $23 \pm 5.4$ (Figure 1). Recurrent disease occurred in four patients (9.5\%) during 66 months of median follow-up. The disease-free and overall survival of the entire patients were 64 and 66 months, respectively. There was no cancer-related death in this series.

\section{Real-time PCR of Leptin and Leptin-R}

Leptin and Leptin- $\mathrm{R}$ were expressed in all patients. The average ratios of $\mathrm{Ct}$ value of Leptin versus reference and Leptin- $\mathrm{R}$ versus reference were $1 \pm 0.08$ and $1 \pm 0.07$, respectively (Figure $2 \mathrm{a}, 2 \mathrm{~b}$ ).

\section{Relationship between clinical data and the expressions of Leptin and Leptin-R}

Table 2 shows the relationships between each clinical parameter and ratio of $\mathrm{Ct}$ value of Leptin and Leptin- $\mathrm{R}$ and reference $\mathrm{Ct}$ value ( $\beta$-actin). There was a significant relationship between the ratio of Leptin Ct value and reference Ct value and BMI $(R=0.39, p<0.01$ Figure 3a), Furthermore, there was a significant relationship between the ratio of Leptin- $\mathrm{R} C t$ value and reference $\mathrm{Ct}$ value and BMI $(\mathrm{R}=0.42, \mathrm{p}<0.005$, Figure $3 \mathrm{~b})$.

There were no significant relationships among the ratio of Leptin and Leptin-R and age, gender, tumor size, lymph node involvement, extra thyroidal infiltration, recurrence and survival.

\section{Discussion}

Obesity is known as a distinct risk factor for the initiation and/ or progression of several diseases and results in comorbidity in some diseases. Some reports reveal BMI to be a risk factor for thyroid cancer. In 2012, Peterson et al. systematically reviewed Relative Risk (RR); 1.1-2.3 for males and 1.0-7.4 for females, in 22 previous reports [8]. In 2014, Xu et al. analyzed the relationship between $\mathrm{BMI}$ and thyroid cancer risk by reviewing three case control studies. 
Table 2: Relationship between Clinical Data and the Expression of Leptin and Leptin-R.

\begin{tabular}{|c|c|c|c|c|c|}
\hline & & Leptin & $\mathrm{p}$ value & Leptin-R & $p$ value \\
\hline Age & & $R=-0.04$ & $p=0.8057$ & $R=0.16$ & $p=0.3001$ \\
\hline \multirow{2}{*}{ Gender } & Make & 1. & \multirow{2}{*}{ p-0.418 } & 1. & \multirow{2}{*}{ p-0.931 } \\
\hline & Female & 1. & & 1. & \\
\hline Tumor size & & $R=-0.17$ & $p=0.2882$ & $R=0.023$ & $p=0.8863$ \\
\hline \multirow{2}{*}{$\begin{array}{l}\text { Lymph node } \\
\text { involvement }\end{array}$} & present & 1. & \multirow{2}{*}{$p-0.508$} & 1. & \\
\hline & absent & 1. & & $p=0.2191 .17$ & \\
\hline \multirow{2}{*}{ Extrathyroidal infiltration } & present & 1. & \multirow{2}{*}{$\mathrm{p}-0.571$} & 1. & \\
\hline & absent & 1. & & p-0.552 1.21 & \\
\hline Survival & & $R=-0.18$ & $p=0.2615$ & $R=0.19$ & $p=0.2163$ \\
\hline \multirow{2}{*}{ Recurrence } & present & 1. & \multirow{2}{*}{$\mathrm{p}-0.54$} & 1. & \multirow{2}{*}{ p-0.247 } \\
\hline & absent & 1. & & 1. & \\
\hline BMI & & $\mathrm{R}=0.39$ & $p<0.01$ & $\mathrm{R}=0.42$ & $\mathrm{p}<0.005$ \\
\hline
\end{tabular}
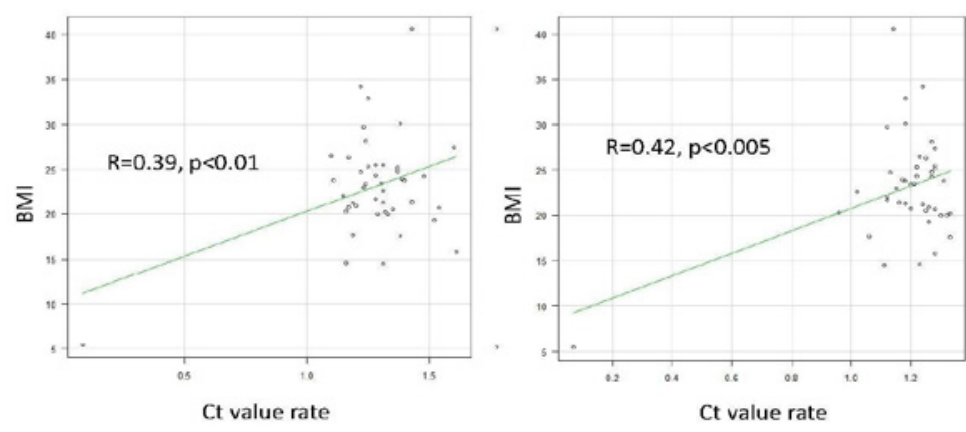

Figure 3: Relationship between BMI and ratio of Ct value of leptin and leptin-R against reference.

There was a significant relationship between the ratio of leptin $\mathrm{Ct}$ value and reference $\mathrm{Ct}$ value and $\mathrm{BMI}(\mathrm{R}=0.39$, $p<0.01 \mathrm{Figure} 3 \mathrm{a})$. There was also a significant relationship between the ratio of leptin- $R$ Ct value and reference $C t$ value and $B M I(R=0.42, p<0.005$, Figure $3 b)$.

They found significant relationships between high BMI and thyroid cancer incidence. Moreover, the Odds Ratio (OR) was 1.72 and the 95\% Confidence Interval (95\% CI) was 1.48-2.00 for patients with high BMI $\left(25-29.9 \mathrm{~kg} / \mathrm{m}^{2}\right)$, and 4.17 and $3.41-5.10$ above $30 \mathrm{~kg} / \mathrm{m}^{2}$ [9]. In contrast, no relationship between BMI and thyroid cancer incidence was found in 1,624,310 subjects in Israel [10]. In general, a higher BMI carries a higher risk for thyroid cancer. Our findings demonstrated a positive correlation between BMI and Leptin and Leptin-R expression on cancer cells. The cause of the correlation was unclear; it may be an initiatory effect of thyroid cancer due to high BMI or by expression of Leptin in thyroid cancer independently of the serum Leptin level.

Leptin is a well-known factor involved in the regulation of body weight and is an important mediator of obesity [2]. Adipose tissue is an important endocrine organ, secreting several hormones including Leptin and adiponectin, and chemokines that can regulate tumor behavior, inflammation, and the tumor microenvironment [11].

The signaling pathways activated by Leptin- $\mathrm{Rb}$ include the classic cytokine Janus kinase 2/signal transducer and activator of transcription 3 (JAK2/STAT3) pathway; the Ras/extracellular signalregulated kinases 1/2 (Ras/ERK1/2) signaling cascade; and the phosphoinositide 3 kinase/protein kinase B (PI3K/Akt) [12]. In vitro, Cheng et al. demonstrated that the PI3K/AKT, JAK/STAT and Map kinase pathways are involved in the growth of thyroid cancer [13]. Other cancers such as prostate [14], breast [15] and hepatocellular carcinoma have been shown to express Leptin and Leptin- $\mathrm{R}$ on their cells [16]. Furthermore, the expression levels and correlations with clinical features in thyroid cancer have been demonstrated. In studies of papillary thyroid carcinoma patients, Leptin-R was found to be expressed in $80.1 \%$ [6], 51.0\% [7], 73.7\% [17], and 57.1\%. The expression ratio of Leptin on cancer cells was reported as $49.1 \%$ [6], $36.7 \%$ [7], 72.4\% [17] and $42.2 \%$. We detected 100\% expressions of Leptin and Leptin-R on thyroid cancer cells (papillary and poorly differentiated thyroid carcinoma). We used real-time PCR for detection, while other studies employed immunohistochemistry. The relationship between clinical features and the expressions of Leptin and Leptin- $\mathrm{R}$ were also demonstrated in other reports. Higher Leptin and Leptin-R expressions were strongly associated with aggressive clinical and pathological features [6]. Some researchers have shown that Leptin and Leptin-R expression are strongly correlated with greater age, larger tumor size [7,17], nodal metastasis [7] and disease free survival. Our findings revealed no clinical correlations with expressions of Leptin and Leptin-R except for BMI.

\section{Conclusion}

$\mathrm{BMI}$ is regulated by the expression of serum Leptin, and a lower serum Leptin level resulted in higher BMI. However, in thyroid 
cancer, higher Leptin and Leptin-R were significantly correlated with high BMI. Further exploration of the relationship between serum Leptin and intratumoral Leptin levels should be undertaken.

\section{Acknowledgment}

This work was supported in part by a grant from the Kawasaki Medical School Study Project 2016 (No. 28-B-2).

I (K Tanaka) disclose receipt of research funding and an honorarium from Eizai Pharma Co. Ltd.

We appreciate Ms. Tsuboi for her support with real-time PCR work.

\section{References}

1. Emily Peterson, Prithwish De, Robert Nuttall. BMI, Diet and Female Reproductive Factors as Risks for Thyroid Cancer: A Systematic Review. PLOS One. 2012; 7: e29177

2. Schwartz MW, Baskin DG, Kaiyala KJ, Woods SC. Model for the regulation of energy balance and adiposity by the central nervous system 1-3. Am J Clin Nutr. 1999; 69: 584-596.

3. Peelman F, Zabeau L, Moharana K, Savvides SN, Tavernier J. Insights into signaling assemblies of the leptin receptor. J Endocrinol. 2014; 223: T9-T23.

4. Oge A, Bayraktar F, Saygili F, Guney E, Demir S. TSH influences serum leptin levels independent of thyroid hormones in hypothyroid and hyperthyroid patients. Endocrine J. 2005; 52: 213-217.

5. Garofalo C, Surmecz E. Leptin and cancer. J Cell Physiol. 2006; 207: 12-22.

6. Uddin S, Bavi P, Siraj AK, Ahmed M, Al-Rasheed M, Hussain AR, et al. leptin- $\mathrm{R}$ and its association with PI3K/AKT signaling pathway in papillary thyroid carcinoma. Endocr Relat Cancer. 2010; 17: 191-202.

7. Cheng SP, Chi CW, Tzcen CY, Yang TL, Lee JJ, Liu TP, et al. Clinicopathologic significance of leptin and leptin receptor expressions in papillary thyroid carcinoma. Surgery. 2010; 147: 847-853.

8. Li Xu, Matthias Port, Stefano Landi, Gemignani F, Cipollini M, Elisei R, et al. Obesity and the Risk of Papillary Thyroid Cancer: A Pooled Analysis of Three Case-Control Studies. Thyroid. 2014; 24: 966-974

9. Alon Farfel, Jeremy D. Kark, Estela Derazne, et al. Predictors for Thyroid Carcinoma in Israel:A National Cohort of 1,624,310 Adolescents Followed for up to 40 Years. Thyroid. 2014; 24: 987-993.

10. VanSaum MN. Molecular pathways: adiponectin and leptin signaling in cancer. Clin Cancer Res. 2013; 19: 1926-1932.

11. Sweeney G. Leptin signalling. Cell Signal. 2002; 14: 655-663.

12. Cheng SP, Y PH, Hsu YC, Cahng YC, Huang SY, Lee JJ, et al. Leptin enhances migration of human papillary thyroid cancer cells through the PI3K/ AKT and MEK/ERK signaling pathways. Oncol Rep. 2011; 26: 1265-1271.

13. Saglam K, Aydur E, Yilmaz M, Goktas S. Leptin influences cellular differentiation and progression in prostate cancer. J Urol. 2003; 169:13081311.

14. Ishikawa M, Kitayama J, Nagawa H. Enhanced expression of leptin and leptin receptor (OB-R) in human breast cancer. Clin Cancer Res. 2004; 10 4325-4331.

15. Wang XJ, Yuan SL, Lu Q, et al. Potential involvement of leptin in carcinogenesis of hepatocellular carcinoma. World J Gastroenterol. 2004; 10: $2478-2481$.

16. Zhang GA, Hou S, Zhou SJ, Wang $X$ and Cui W. Clinicopathological implications of leptin and leptin receptor expression in papillary thyroid cancer. Oncol Let. 2013; 5: 797-800.

17. Fan YL, Li XQ. Expression of leptin and its receptor in thyroid carcinoma: distinctive prognostic significance in different subtypes. Clinical Endocrinology. 2015; 83: 261-267.
Annals Thyroid Res - Volume 4 Issue 1 - 2018

Submit your Manuscript | www.austinpublishing group.com

Katsuhiro et al. () All rights are reserved
Citation: Katsuhiro T, Mai S, Shiori S, Ryohei O, Wataru S and Yusuke O. Expression of Leptin and Leptin Receptor Messenger Ribonucleic Acids in Thyroid Carcinoma. Annals Thyroid Res. 2018; 4(1): 126-129. 